\title{
Demyelinating myelitis vs Acute ascending necrotizing myelitis
}

\begin{abstract}
We present the case of a 26 year old male that was diagnosed with an acute ascending necrotizing myelitis, the initial symptoms were oppressive pain in the chest and numbness in both hands, followed by weakness of the arms and legs. The MRI detected a hyperintensity from $\mathrm{C} 4$ to $\mathrm{T} 1$. We suspected a demyelinating event, despite the prompt intervention the patient had progression of the disease, showing an aggressive disease. This case reveals a disease that mimics acute demyelinating diseases.
\end{abstract}

Keywords: acute necrotizing myelitis, ascending necrotizing myelitis, necrotizing myelitis
Volume II Issue 4 - 202I

\author{
Jaime Adolfo Luna Martìnez,' Elida Moran \\ Guel, ${ }^{2}$ Nadia Viviana Sandoval Rodríguez, ${ }^{3}$ \\ Jorge Alberto Gonzalez Lucio ${ }^{4}$ \\ 'Neurology, Universidad Autonoma de Coahuila/ Unidad Medica \\ de Alta Especialidad No. 7I, Mexico \\ ${ }^{2}$ Intensive Care Unit, Universidad Autonoma de Coahuila / \\ Unidad Medica de Alta Especialidad No. 7I, Mexico \\ ${ }^{3}$ Internal Medicine, Universidad Autonoma de Coahuila/ Unidad \\ Medica de Alta Especialidad No. 7I, Mexico \\ ${ }^{4}$ Radiologia, Universidad Autonoma de Sinaloa/ Hospital General \\ de Sinaloa, Culiacan Sinaloa, Mexico
}

\author{
Correspondence: Jaime Adolfo Luna Martinez, Neurology, \\ Universidad Autonoma de Coahuila, UMAE No. 7I, Blvd. \\ Revolución y Calle 27 S/N Centro Torreon Jardin, Torreón, \\ Coahuila, Mexico,Tel 87I-729-0800,Email drluna@outlook.es \\ Received: July II, 202I | Published: August 24, 202 I
}

Abbreviations: ANM, acute necrotizing myelitis; CNS, central nervous system; ANS, autonomic nervous system, MAVS, vascular malformations, $\mathrm{CMV}$, cytomegalovirus

\section{Introduction}

Acute ascending necrotizing myelitis, being a very rare and deadly disease more frequently associated with herpes virus and paraneoplastic syndromes. The clinical signs are a manifestation of an acute spinal cord transverse myelitis, suspicion should be based on a patient with no important risk factors, the physician should be intentionally looking for a previous history of methylphenidate and energy drinks. The treatment options are anticoagulant at a therapeutic dosing. In this report we present the case of an ANM presenting initially an acute chest pain and bilateral hand numbness with no identifiable cause. ${ }^{1,2}$

\section{Case report}

Male patient, 26 years old, traumatology resident with no significant hereditary or family history, who denies chronic degenerative diseases, positive smoking at a rate of 6 cigarettes a day for the last 10 years, smoking rate of 3 packs/year. Diagnosed with depression, in treatment with sertraline one tablet orally every 24 hours.

Patient started his condition on the 13th of April, 2020 in the morning when he presented oppressive-punching pain sensation in the precordial region, intensity $7 / 10$, without irradiation, without other accompanying symptoms, which improved after the administration of buprenorphine, followed by numbness and hands' weakness, predominantly right, subsequent affection of pelvic limbs, evolving with inability to stand and walk.

On admission to the hospital he was found with flaccid quadriparesis, DTR $2 / 4$ in thoracic limbs and $0 / 4$ in pelvic limbs, without frontal liberation signs or objective sensory deficit, he presented hypotension. Negative studies for herpes virus and CMV infectious processes. He also presented acute urinary retention so an urinary catheter was placed. ${ }^{3}$

Spinal MRI showed hyper intense lesions at thoracic level, suggestive of demyelinating process, so it was decided to administer steroid pulses. The lumbar puncture showed normal cytological and cytochemical studies, BOC, immunoglobulin and TORCH studies were also requested and the results were pending. Basic serology tests for lupus as well as general urine examination and chest $\mathrm{X}$-ray were normal.

\section{Neurological examination}

Patient awake, attentive, oriented in person, time and situation, immediate, short, and long term memory without alterations. As for language, he understands, shows fluency, repeats, names, with reading and writing execution without alterations. Judgment, abstraction, and calculation without alterations. Praxias and gnosias without alterations.

Cranial nerves: I: No alterations. II: visual acuity 20/100 bilateral without glasses, 20/20 with glasses, colorimetry, campimetry by confrontation without alterations, fundus by direct ophthalmoscopy not adequate due to poor cooperation of the patient, normal papilla, no exudates were found, vein/artery ratio 3/1. III, IV, VI: no limitation to perform ductions, versions, vergence, pupils of $3 \mathrm{~mm}$, photomotor reflex, consensual and accommodation reflex present. V: Sensory portion preserved in the three branches. Motor portion with adequate trophism, tone and strength of masticatory muscles. VII symmetrical gesticulation, taste recognition is deferred in $2 / 3$ of the tongue. VIII: normo-hearing attitude. IX, X: central uvula, symmetrical palate, gag reflex present, perception and recognition of flavors deferred. XI: adequate tone, trophism and strength of sternocleidomastoid and trapezius muscles. XII: adequate tongue mobility. ${ }^{4,5}$

Motor system: tone and trophism preserved, strength: right proximal thoracic extremity $4 / 5$ and distal $3 / 5$, left proximal $4 / 5$ and 
distal $3 / 5$. Right proximal pelvic extremity $4 / 5$ and distal $1 / 5$, left proximal $4 / 5$ and distal $1 / 5$.

Muscle stretch reflexes: upper extremities trapezius $+/++$, bicipital $++/++++$, styloradialis $++/++++$, lower extremities patellar $+/++++$, Achilles $0 /++++$, flexor plantar response, Trömner and Hoffman absent.

It was decided to administer methylprednisolone pulses with which he presented a favorable clinical response with improvement in the strength of the four limbs. It was decided to discharge him home on April 19, 20 due to clinical improvement, studies of BOC, immunoglobulins and TORCH were negative.

2 days after his discharge, he referred morning pain at a cervical level, intensity $5 / 10$, he was medicated with ibuprofen and hot compresses, with which he presented partial improvement, however the pain increased in intensity, in addition to being accompanied by peribuccal cyanosis and neurological deficit, so he was transferred back to the hospital. On admission to the emergency room he was found with oxygen desaturation, with flaccid quadriparesis, REMS $2 / 4$ in thoracic limbs, $0 / 4$ in pelvic limbs, no abnormal reflexes, no objective sensory deficit, having hypotension, negative laboratory studies for infectious process. A new MRI was performed (Figure 1), which showed vascular enhancement, the angiotomography didn't show any abnormality so the spinal cord lesions correspond to transverse myelitis, in contrast with data of abnormal enhancement in the upper region of the spinal cord.

The patient presented cardiorespiratory arrest which responded to the first cycle of cardiopulmonary resuscitation with return of spontaneous circulation and sinus rhythm. Advanced airway management was performed urgently and the patient was transferred to the intensive care unit. A transthoracic echocardiogram was performed in which data of aneurysm with valvular involvement was found, in addition to a small pericardial effusion of $7 \mathrm{~mm}$ in the posterior region, without hemodynamic compromise, so angiotomography or angioresonance was requested to rule out ischemic cause. Patient continued with $0 / 5$ strength in all extremities, but with preserved sensitivity and areflexia. Patient continued with persistent fever and developed health care-associated pneumonia. ${ }^{3}$

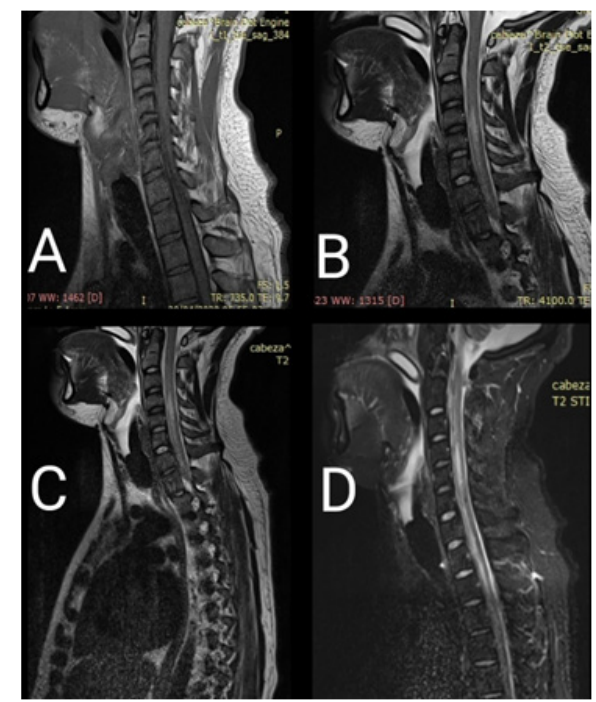

Figure I Magnetic resonance imaging (MRI) (A) Sagittal TIWI Showing an abnormal hypodensity in C5 level. (B) and (C) Sagittal T2WI showing an hyperintense signal in the spinal cord with a length of 4 vertebral levels. (D) A midsagittal T2STIR of the spinal cord shows central hyperintense signal changes from cervical to the upper thoracic levels.
The patient presented with D-dimer elevation up to 2500. Due to suspicion of vascular cause either thrombotic, aneurysmal, vasculitis, compressive traumatic injury or atherosclerosis, of the previous spinal syndrome, studies were requested to address vasculitis, as well as angiotomography, and anticoagulation therapy was initiated. Angiotomography ruled out vascular causes of the patient's disorder. ${ }^{5-7}$

The patient continued with generalized hypotonia and tetraplegia, without progression or improvement of the motor deficit, remaining dependent on mechanical ventilation, without presence of spontaneous breathing.

The patient, since his second hospital admission presented elevated transaminases, on $04 / 27 / 20$ with an increase of 2.8 times over normal values of ALT and AST of 2.2 times, with bilirubin levels below 2, normal coagulation exams, he also presented elevated creatine kinase and $\mathrm{MB}$ creatine kinase, so he was evaluated to rule out rhabdomyolysis and cardiac ischemia. PCR for Sars-CoV2 was negative.

On 04/30/20 CSF studies were reported: negative for oligoclonal bands, negative for Beta 2 glycoprotein, negative P-ANCA and C-ANCA, negative anti aquaporin 4 and negative anti MOG. The patient continued with deterioration and on $05 / 03 / 20$ presented oxygen desaturation, without improvement with conventional ventilation method, so it was decided to administer double sedation in addition to vecuronium as muscle relaxant, he was placed in prone position and changed to APRV ventilation mode, cultures were taken and antibiotics were adjusted.

The patient continued with deterioration, adding hypotension to the picture, which did not improve with the use of vasopressors, in addition to continuing with respiratory deterioration. The patient presented cardiac arrest on $05 / 06 / 20$, cardiopulmonary resuscitation maneuvers were started, without return to spontaneous circulation, and death was declared at 12:00 hrs.

Some days later, colleagues referred to us that the patient had ingested amphetamines and a at least 3 energy drinks per day, due to the spontaneous apparition of the disease without previous clinical record, fast progression of the disease and the lack of response to an immunomodulatory treatment we diagnosed Acute Ascending Necrotizing Myelitis.

\section{Discussion}

Patients that arrive with an acute medullary syndrome should be evaluated by a Neurologist because the ANM could be mistaken for a CNS demyelinating condition. After the ANM diagnosis there should be an intentional search for an underlying cause like drugs, MAVS and paraneoplastic causes. This case showed that in patients with minor improvement after immunomodulatory treatment the diagnosis of ANM should be considered.

\section{Conclusion}

The ANM is a medical condition that has a high mortality and morbidity, the survival rate depends on the time of diagnosis, so it is an obligation for the neurologist to know this condition and its causes.

\section{Acknowledgments}

None.

\section{Conflicts of interest}

The authors declare that there are no conflicts of interest regarding the publication of this paper. 


\section{References}

1. Jacob A, Weinshenker BG. An approach to the diagnosis of acute transverse myelitis. Semin Neurol. 2008;28(1):105-120.

2. Mlsumi H, Ishibashi H, Kanayama K, et al. Necrotizing Myelopathy Associated with Hepatocellular Carcinoma. Jpn J Med. 1988;27(3):333336.

3. Papageorgiou E, Psychogios K, Tzanetakos D, et al. Serial magnetic resonance imaging of acute necrotizing myelitis. $J$ Neurol Sci. 013;333:e666-667.

4. Mirich DR, Kucharczyk W, Keller MA, et al. Subacute necrotizing myelopathy: MR imaging in four pathologically proved cases. $A m J$ Neuroradiol. 1991;12(6):1077-1083.
5. Iwamasa T, Yoshitake H, Sakuda H, et al. Acute ascending necrotizing myelitis in Okinawa caused by herpes simplex virus type 2. Virchows Arch A Pathol Anat Histopathol. 1991;418(1):71-75.

6. Habek M, Adamec I, Pavliša G, et al. Diagnostic approach of patients with longitudinally extensive transverse myelitis. Acta Neurol Belg. 2012;112(1):39-43.

7. Bordignon $\mathrm{KC}$, Montú MB, Ramina R, Arruda WO. Foix-Alajouanine syndrome: Case report. Arq Neuropsiquiatr. 2005;63(2 B):527-529.

8. Ankur Nandan V, Nilesh K, Dibyaranjan B, et al. Acute Transverse Myelitis (Ascending Myelitis) as the Initial Manifestation of Japanese Encephalitis: A Rare Presentation. Case Rep Infect Dis. 2013;2013:1-3. 